\title{
Dynamics of a cable with an attached sliding
}

\author{
mass
}

\section{P. Williams*}

(Received 25 October 2005; revised 23 June 2006)

\begin{abstract}
I describe the development of a three dimensional numerical model of a cable system with an interacting sliding mass. The cable is modelled using a lumped parameter approximation, where the cable is discretised into a collection of viscoelastic point mass elements articulated by frictionless hinges. The sliding mass is modelled as a point mass which is free to move in all three dimensions. The dynamics of the sliding mass are formulated to take into account the constraint provided by the cable, and incorporates a variety of active forces including friction and air drag.
\end{abstract}

\section{Contents}

\section{Introduction}

*School of Aerospace, Mechanical, and Manufacturing Engineering, RMIT University, Bundoora, Australia. mailto:paul.williams@rmit.edu.au

See http://anziamj.austms.org.au/V47EMAC2005/Williams for this article, (C) Austral. Mathematical Soc. 2006. Published July 7, 2006. ISSN 1446-8735 
2 Mathematical model for cable $\quad$ C89

2.1 Tension forces . . . . . . . . . . . . . . . . . C90

2.2 Damping forces . . . . . . . . . . . . . . . . . . C90

2.3 Aerodynamic forces . . . . . . . . . . . . . . . . . C91

2.4 Gravitational forces . . . . . . . . . . . . . . . C92

3 Sliding mass element $\quad$ C92

3.1 Searching algorithm . . . . . . . . . . . . C94

4 Numerical results $\quad$ C95

5 Conclusions $\quad$ C99

$\begin{array}{ll}\text { References } & \text { C100 }\end{array}$

\section{Introduction}

Cable systems arise in many practical applications, such as bridges, underwater systems, aircraft decoy systems, and tethered satellite systems. Methods for approximating the cable motion and its equilibria have been studied for many years. Cable-body systems have been modelled using continuum models based on partial differential equations for strings, as well as lumped parameter representations. In complex applications, the lumped parameter representation is usually the preferred choice for detailed simulation work [1]. Although continuum models are typically considered more accurate, the distinction between the two types of models becomes blurred during numerical simulation when partial derivatives are approximated by means of a finite difference method, or when the system motion is expanded in terms of admissible functions. The lumped parameter representation is the most convenient choice for a variety of cable systems and will be employed in this work. One advantage of the lumped parameter representation is that geometric 
nonlinearities are inherently captured, and situations involving possible loss of tension are easily incorporated.

We focus on the case where a cable is carrying a load which is free to move due to the presence of applied external forces. There are a variety of useful applications where such a model is needed for analysis, such as cable trams, rocket propelled trolleys, or transport of cargo between two ships. Dynamical models of such scenarios have been developed in related work. Wu and Chen [2] used an updated Lagrangian and finite element method to model the cable dynamics with a moving mass particle. They obtained numerical solutions using direct Newmark integration. Al-Qassab et al. [3] derived the equations of motion for a cable carrying a moving particle using Hamilton's principle. Aerodynamic drag and frictional forces were neglected. Tadjbakhsk and Wang [4] also studied the vibrations of a cable with an accelerating mass, but took into account friction and a propulsive force applied to the trolley. They used Galerkin's method to discretize the dynamics. An alternative approach based on a finite element formulation was presented by Zhou et al. [5], who introduced a special "sliding" element to capture the effect of the cable sliding over a point. Friction was ignored in the model, and it was applied in the simulation of a parachute system.

I present a cable model with a sliding mass using a lumped parameter representation. First, the general representation of the cable model is given. The method for incorporating the sliding mass is developed so that the model can be applied with or without a sliding mass. A range of realistic forces on the slider and cable are included, such as air drag, friction, and damping. A simple method for determining the "active" cable segment is outlined, which is used at each time step to locate the element affected by the sliding mass. Finally, numerical simulations illustrate the versatility of the model. 


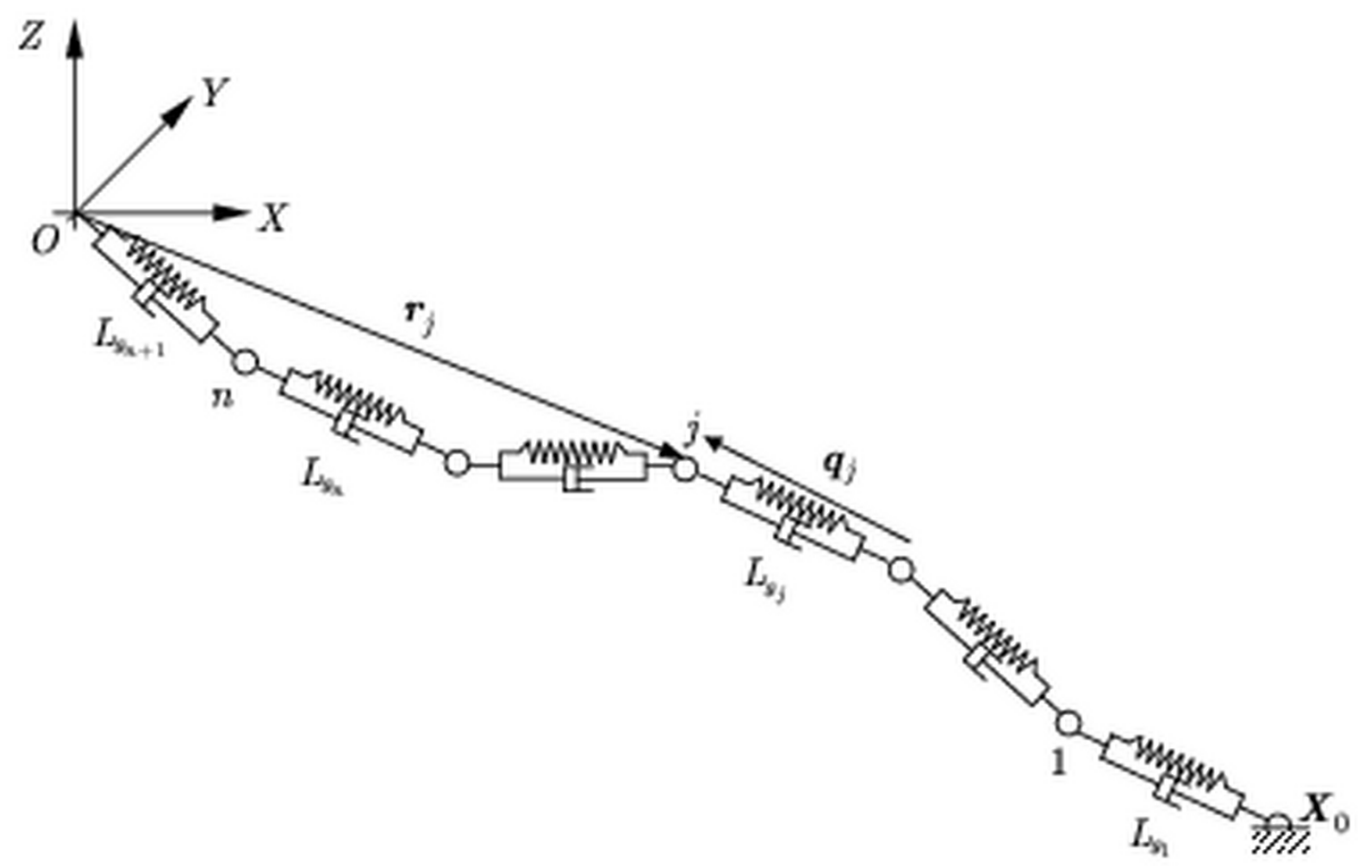

FiguRE 1: Lumped parameter representation of cable object.

\section{Mathematical model for cable}

For the purposes of simplicity, the cable model is derived for a single cable object. The cable is modelled by physically discretising it into a sequence of point mass elements interconnected via viscoelastic springs, as shown in Figure 1. It is assumed that the cable is perfectly flexible, and as a consequence, the point masses act as frictionless hinges. The elements are numbered from 0 at the "free" end, through to $n$ at the "fixed" end. Note that for the results in this paper, the 0th element is fixed. However, in general the 0th mass may be subject to its own dynamical equations or specified kinematic motion. Furthermore, the origin of the cable coordinate system $(0, X, Y, Z)$ may be either fixed or moving with a prescribed motion. 
The dynamical equations for the cable are derived via Newton's second law using the inertial coordinates of the masses. Hence

$$
m_{j} \ddot{\mathbf{r}}_{j}=\mathbf{F}_{j}^{\mathrm{s}}+\mathbf{F}_{j}^{\mathrm{d}}+\mathbf{F}_{j}^{\mathrm{g}}+\mathbf{F}_{j}^{\mathrm{drag}}, \quad j=1, \ldots, n,
$$

where $\mathbf{r}_{j}=x_{j} \mathbf{i}+y_{j} \mathbf{j}+z_{j} \mathbf{k}$, the elemental mass of the $j$ th cable segment is

$$
m_{j}=\frac{1}{2} \rho A\left(L_{s_{j+1}}+L_{s_{j}}\right), \quad j=1, \ldots, n,
$$

$\rho$ is the density of the cable material, $A$ is the cross-sectional area, and $L_{s_{j}}$ is the unstrained length of the $j$ th cable segment. The external forces on the right-hand side of Equation (1) are due to tension/spring forces, damping, aerodynamic drag, and gravity, denoted by the superscripts s, d, drag, and g, respectively. These forces are defined in the following subsections.

\section{$2.1 \quad$ Tension forces}

Consider the strain in the $j$ th element,

$$
\varepsilon_{j}= \begin{cases}\left(\left|\mathbf{q}_{j}\right|-L_{s_{j}}\right) / L_{s_{j}}, & \left|\mathbf{q}_{j}\right| \geq L_{s_{j}} \\ 0, & \left|\mathbf{q}_{j}\right|<L_{s_{j}}\end{cases}
$$

where $L_{s_{j}}$ is the unstrained segment length. The tension force is defined by Hooke's law as $T_{j}=E A \varepsilon_{j}$, and hence the total force on the $j$ th mass is

$$
\mathbf{F}_{j}^{\mathrm{s}}=T_{j+1} \frac{\mathbf{q}_{j+1}}{\left|\mathbf{q}_{j+1}\right|}-T_{j} \frac{\mathbf{q}_{j}}{\left|\mathbf{q}_{j}\right|},
$$

where $\mathbf{q}_{j}=\left(x_{j}-x_{j-1}\right) \mathbf{i}+\left(y_{j}-y_{j-1}\right) \mathbf{j}+\left(z_{j}-z_{j-1}\right) \mathbf{k}$.

\subsection{Damping forces}

The damping forces in the cable are assumed to be proportional to the strain rate. Express the length of the $j$ th segment as

$$
l_{j}=L_{s_{j}}\left(1+\varepsilon_{j}\right) .
$$


Taking the derivative of Equation (5) and rearranging gives

$$
\dot{\varepsilon}_{j}=\frac{\dot{l}_{j}}{L_{s_{j}}}-\frac{\dot{L}_{s_{j}}}{L_{s_{j}}}\left(1+\varepsilon_{j}\right) .
$$

Using $i_{j}=\dot{\mathbf{q}}_{j} \cdot \mathbf{q}_{j} /\left|\mathbf{q}_{j}\right|$ and $l_{j}=\left|\mathbf{q}_{j}\right|$, the strain rate

$$
\begin{aligned}
\dot{\varepsilon}_{j}= & \frac{1}{L_{s_{j}} \sqrt{\left(x_{j}-x_{j-1}\right)^{2}+\left(y_{j}-y_{j-1}\right)^{2}+\left(z_{j}-z_{j-1}\right)^{2}}} \times \\
& \times\left[\left(x_{j}-x_{j-1}\right)\left(\dot{x}_{j}-\dot{x}_{j-1}\right)+\left(y_{j}-y_{j-1}\right)\left(\dot{y}_{j}-\dot{y}_{j-1}\right)\right. \\
& \left.\quad+\left(z_{j}-z_{j-1}\right)\left(\dot{z}_{j}-\dot{z}_{j-1}\right)\right] \\
& -\frac{\dot{L}_{s_{j}}}{L_{s_{j}}^{2}} \sqrt{\left(x_{j}-x_{j-1}\right)^{2}+\left(y_{j}-y_{j-1}\right)^{2}+\left(z_{j}-z_{j-1}\right)^{2}} .
\end{aligned}
$$

Hence, the damping force is defined by

$$
\mathbf{F}_{j}^{\mathrm{d}}=D_{j+1} \frac{\mathbf{q}_{j+1}}{\left|\mathbf{q}_{j+1}\right|}-D_{j} \frac{\mathbf{q}_{j}}{\left|\mathbf{q}_{j}\right|},
$$

where

$$
D_{j}= \begin{cases}C \dot{\varepsilon}_{j}, & \left|\mathbf{q}_{j}\right| \geq L_{s_{j}}, \\ 0, & \left|\mathbf{q}_{j}\right|<L_{s_{j}},\end{cases}
$$

and $C$ is the damping constant of the cable material.

\subsection{Aerodynamic forces}

The aerodynamic drag forces on the cable are calculated by ignoring the skin friction component of the drag. The component of the normal velocity for the $j$ th mass is

$$
\mathbf{v}_{j}^{\mathrm{n}}=\mathbf{v}_{j}-\frac{1}{2} \mathbf{v}_{j} \cdot\left(\frac{\mathbf{q}_{j}}{\left|\mathbf{q}_{j}\right|}+\frac{\mathbf{q}_{j+1}}{\left|\mathbf{q}_{j+1}\right|}\right)
$$


which averages the projected velocity components based on the orientation of the adjacent cable segments. The drag force based on the normal component of velocity is

$$
\mathbf{F}_{j}^{\mathrm{drag}}=-\frac{1}{2} \rho_{j}^{\mathrm{air}} L_{s_{j}} d C_{D} \mathbf{v}_{j}^{\mathrm{n}}\left|\mathbf{v}_{j}^{\mathrm{n}}\right|,
$$

where $d$ is the cable diameter, $C_{D}$ is the normal drag coefficient (taken to be 1.1), and $\rho_{j}^{\text {air }}$ is the air density at the $j$ th mass.

\subsection{Gravitational forces}

A uniform gravitational field in the vicinity of the cable provides the force

$$
\mathbf{F}_{j}^{\mathrm{g}}=-m_{j} g \mathbf{k} .
$$

\section{$3 \quad$ Sliding mass element}

In order to include the effect of a freely sliding mass, it is necessary to treat the slider $m_{s}$ as an independent particle governed by

$$
m_{s} \ddot{\mathbf{r}}_{s}=\mathbf{F}_{s}^{\mathrm{N}}+\mathbf{F}_{s}^{\mathrm{f}}+\mathbf{F}_{s}^{\mathrm{g}}+\mathbf{F}_{s}^{\mathrm{drag}},
$$

where $m_{s}$ is the mass of the sliding particle, and the external forces acting on the particle are due to the normal reaction of the cable on the particle, friction forces, gravity and drag, denoted by the superscripts N, f, g and drag, respectively. For simplicity, the particle is assumed to be in contact with the cable at a point so that the slope of the cable across the particle's position is discontinuous in the general case. For convenience, the "active" cable segment is defined to be the one that the slider is currently in contact with. Figure 2 shows a general depiction of an active sliding element. To model the reaction of the cable on the slider and the friction forces, a normal tangential coordinate system is employed $\left(\mathbf{n}, \mathbf{t}, \mathbf{k}_{N}\right)$. 


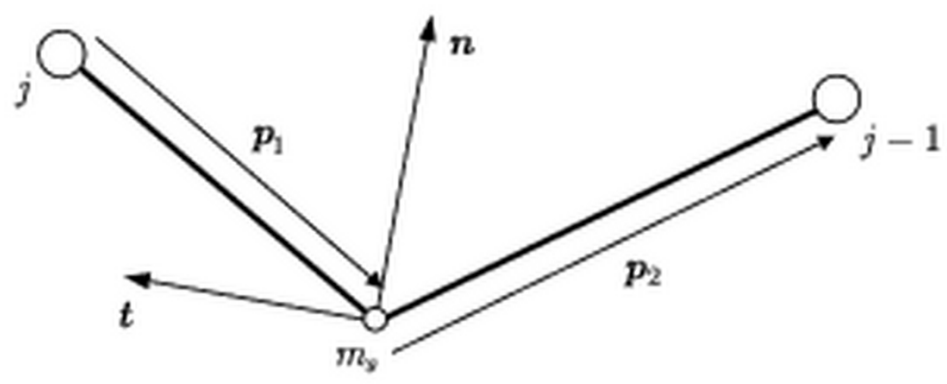

FIGURE 2: Sliding mass element.

The osculating plane of the normal tangential frame is defined via the vector $\mathbf{k}_{N}=\mathbf{p}_{1} \times \mathbf{p}_{2}$. The normal vector to the cable at the particle's position is defined to bisect the angle between the two cable segments

$$
\mathbf{n}=\frac{1}{2}\left[\frac{\mathbf{k}_{N} \times \mathbf{p}_{1}}{\left|\mathbf{k}_{N} \times \mathbf{p}_{1}\right|}+\frac{\mathbf{k}_{N} \times \mathbf{p}_{2}}{\left|\mathbf{k}_{N} \times \mathbf{p}_{2}\right|}\right] .
$$

Finally, a vector tangent to the cable at the contact point of the particle is defined as

$$
\mathbf{t}=\frac{\mathbf{k}_{N} \times \mathbf{n}}{\left|\mathbf{k}_{N} \times \mathbf{n}\right|} .
$$

The presence of the slider on the active segment deforms the segment to produce the modified tension value

$$
T_{s}=\frac{E A}{L_{s_{j}}^{*}}\left(\left|\mathbf{p}_{1}\right|+\left|\mathbf{p}_{2}\right|-L_{s_{j}}^{*}\right) .
$$

The tension is assumed to be constant across the active cable segment, and the effect of friction is applied to the adjacent lumped masses. The modified tension value replaces the tension force applied to the $j$ th and $(j-1)$ th masses as given in Equation (4).

The normal force of the cable on the slider is determined by the components of the tension force

$$
\mathbf{T}_{1}=-T_{s} \mathbf{p}_{1} /\left|\mathbf{p}_{1}\right|, \quad \mathbf{T}_{2}=T_{s} \mathbf{p}_{2} /\left|\mathbf{p}_{2}\right|,
$$


projected onto the direction of the normal vector, that is,

$$
\mathbf{F}_{s}^{\mathrm{N}}=\left(\mathbf{T}_{1} \cdot \mathbf{n}+\mathbf{T}_{2} \cdot \mathbf{n}\right) \mathbf{n} .
$$

Note: if the tension becomes zero, no normal force is exerted on the slider.

Frictional forces are assumed to act parallel to the tangent vector t. Define the sum of external forces acting on the slider (except the normal force and friction) as $\mathbf{F}_{\text {ext }}$. The maximum frictional force that can be exerted by the cable is governed by $F_{\max } \equiv \mu_{k}\left|\mathbf{F}_{s}^{\mathrm{N}}\right|$. Cases where the slider is in motion along the tangent direction and when it is stationary must be distinguished. In general, the friction force is governed by

$$
\mathbf{F}_{s}^{\mathrm{f}}= \begin{cases}-\operatorname{sign}\left(\mathbf{v}_{s} \cdot \mathbf{t}\right) F_{\max } \mathbf{t}, & \left|\mathbf{F}_{\text {ext }} \cdot \mathbf{t}\right| \geq F_{\max }, \\ -\operatorname{sign}\left(\mathbf{v}_{s} \cdot \mathbf{t}\right)\left|\mathbf{F}_{\text {ext }} \cdot \mathbf{t}\right| \mathbf{t}, & \left|\mathbf{F}_{\text {ext }} \cdot \mathbf{t}\right|<F_{\max }\end{cases}
$$

The direction of the frictional force in the case where the slider has no velocity component along the cable tangent direction is governed by the sign of the projection $\mathbf{F}_{\text {ext }} \cdot \mathbf{t}$. The frictional forces of the slider on the cable are equal and opposite, and are applied to the adjacent lumped masses according to

$$
\mathbf{F}_{j}^{\mathrm{f}}=\frac{\left|\mathbf{p}_{2}\right| \mathbf{F}_{s}^{\mathrm{f}}}{\left|\mathbf{p}_{1}\right|+\left|\mathbf{p}_{2}\right|}, \quad \mathbf{F}_{j-1}^{\mathrm{f}}=\frac{\left|\mathbf{p}_{1}\right| \mathbf{F}_{s}^{\mathrm{f}}}{\left|\mathbf{p}_{1}\right|+\left|\mathbf{p}_{2}\right|} .
$$

Note that these forces are applied by modifying the active tension forces on the cable in Equation (4).

\subsection{Searching algorithm}

As the sliding mass moves along the cable, the active cable segment continually changes and singularities in some of the governing equations occur. For example, consider the case where the slider approaches the position of one of the lumped masses representing the cable in Equation (14). In such a circumstance, one of the denominators in the expression approaches zero. Hence 
care must be taken during implementation of the algorithm to monitor the proximity of the slider to the discrete cable elements. Defining a proximity distance, it is a simple matter to adjust the definition of the appropriate $\mathbf{p}$ to be defined as along the line from $m_{s}$ to $m_{j-2}$ or $m_{j+1}$, depending on its position. When such a situation occurs, it is necessary to adjust the value of $L_{s_{j}}^{*}$ in Equation (16) accordingly.

An effective strategy for determining the active cable element for most practical scenarios is to first compute the values of $\mathbf{p}_{1}$ and $\mathbf{p}_{2}$ for every element, assuming no a priori knowledge of the active cable element from a previous time step. The active cable element is taken to be the one satisfying $\min _{j}|| \mathbf{p}_{1 j}|+| \mathbf{p}_{2 j}\left|-L_{s_{j}}\right|$. This procedure could fail if the cable has loops or if two segments are very close to each other. However, numerical experience suggests that the procedure is adequate for typical sliding configurations.

\section{Numerical results}

To demonstrate the effectiveness of the model, an example of an inclined cable is used [4]. Note that the model was first validated using a much simpler simulation [5]. The following simulation properties are used: $\rho=113575 \mathrm{~kg} / \mathrm{m}^{3}$, $L=3900 \mathrm{~m}, d=5 \mathrm{~mm}, C=50 \mathrm{Ns}, n=100, E=70 \mathrm{GPa}, m_{s}=10193.6 \mathrm{~kg}$. Simulations are performed with and without friction, where $\mu_{k}=0.1$ for the cases with friction. The left support is located at the coordinates $(0,0,0)$, whereas the right support is located at $(4500,0,-750) \mathrm{m}$. The initial conditions of the cable are the static equilibrium conditions for the cable, determined by a nonlinear root-finding method for zero velocity and acceleration of the cable masses. The initial position of the slider is $0.1 \%$ of the distance along the cable element closest to the left support. A variable order, variable step, stiff integrator scheme integrates the equations of motion. Note that not all parameters were given in [4], but the initial configuration tension at the left support of $252 \mathrm{kN}$ correlates well with the $250 \mathrm{kN}$ used in [4]. 


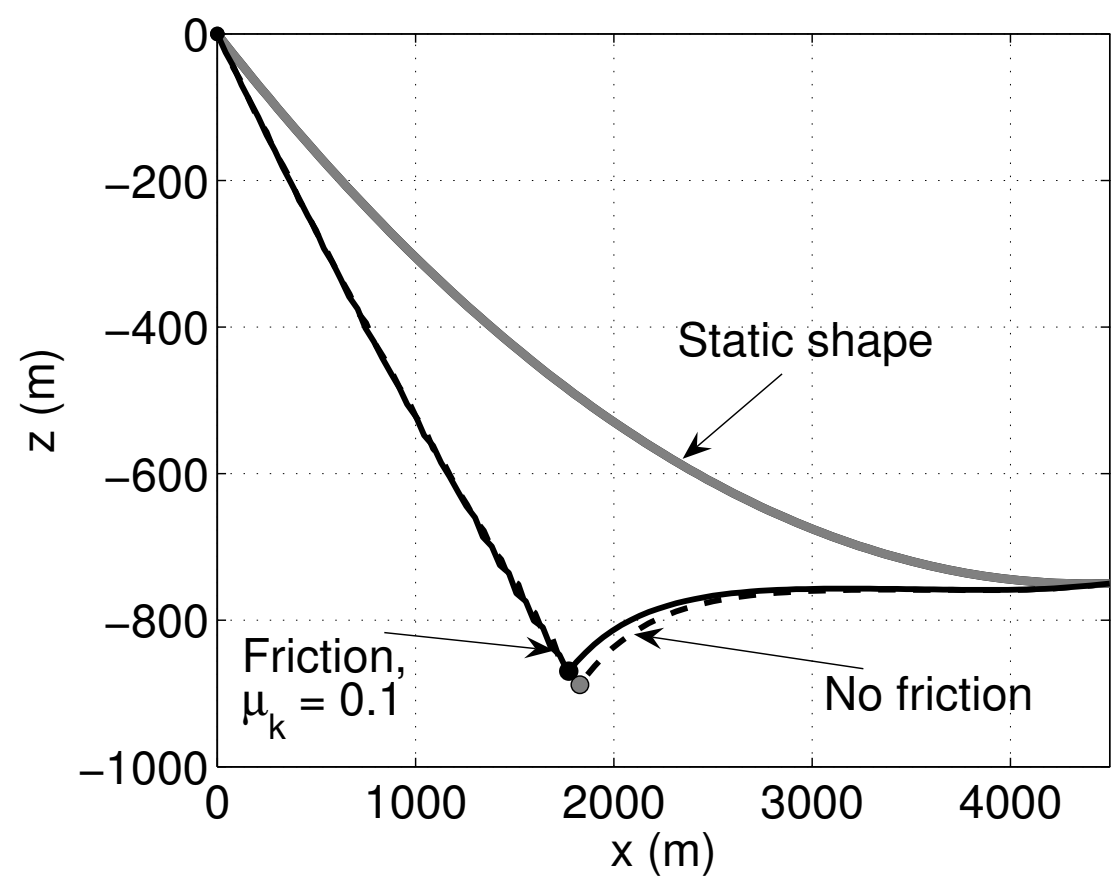

Figure 3: Rocket propelled trolley simulation [4], snapshot at $t=14 \mathrm{sec}$. 


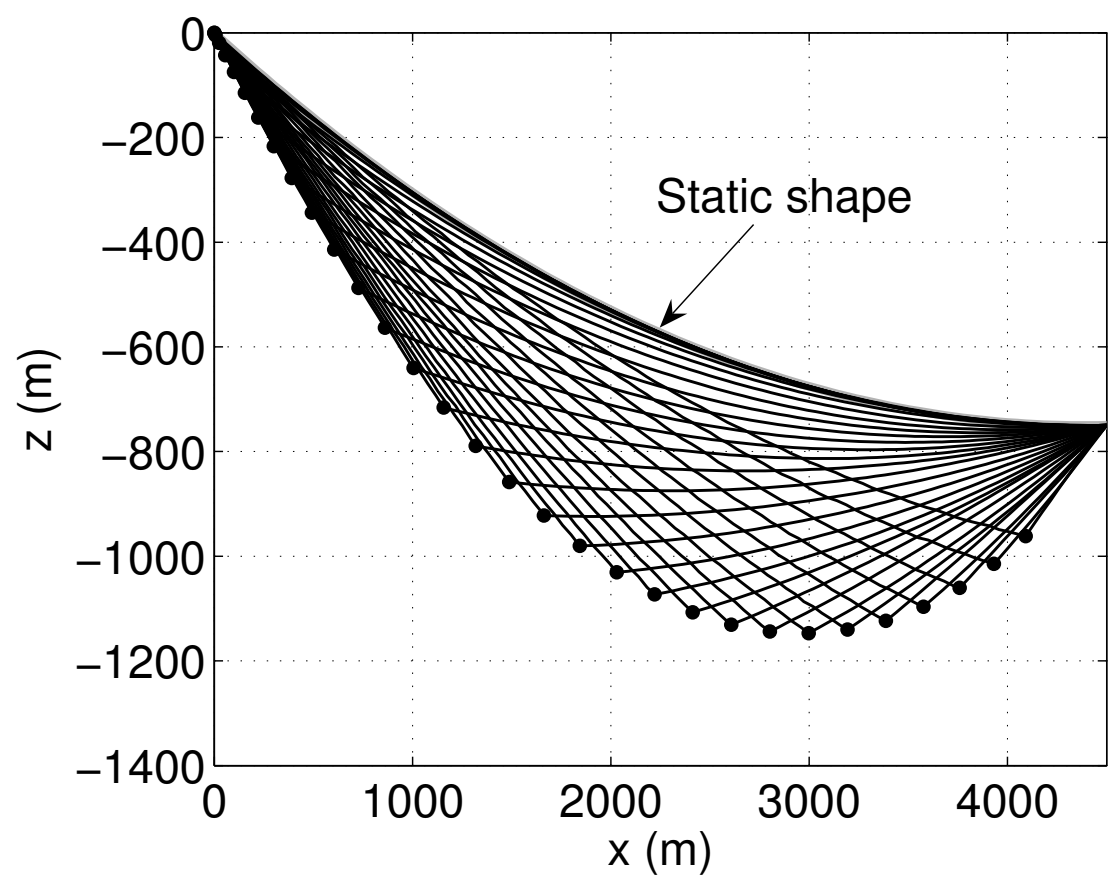

Figure 4: Dynamic and static cable shapes during sliding mass motion shown at $2 \mathrm{sec}$ intervals ( $60 \mathrm{sec}$ total). 


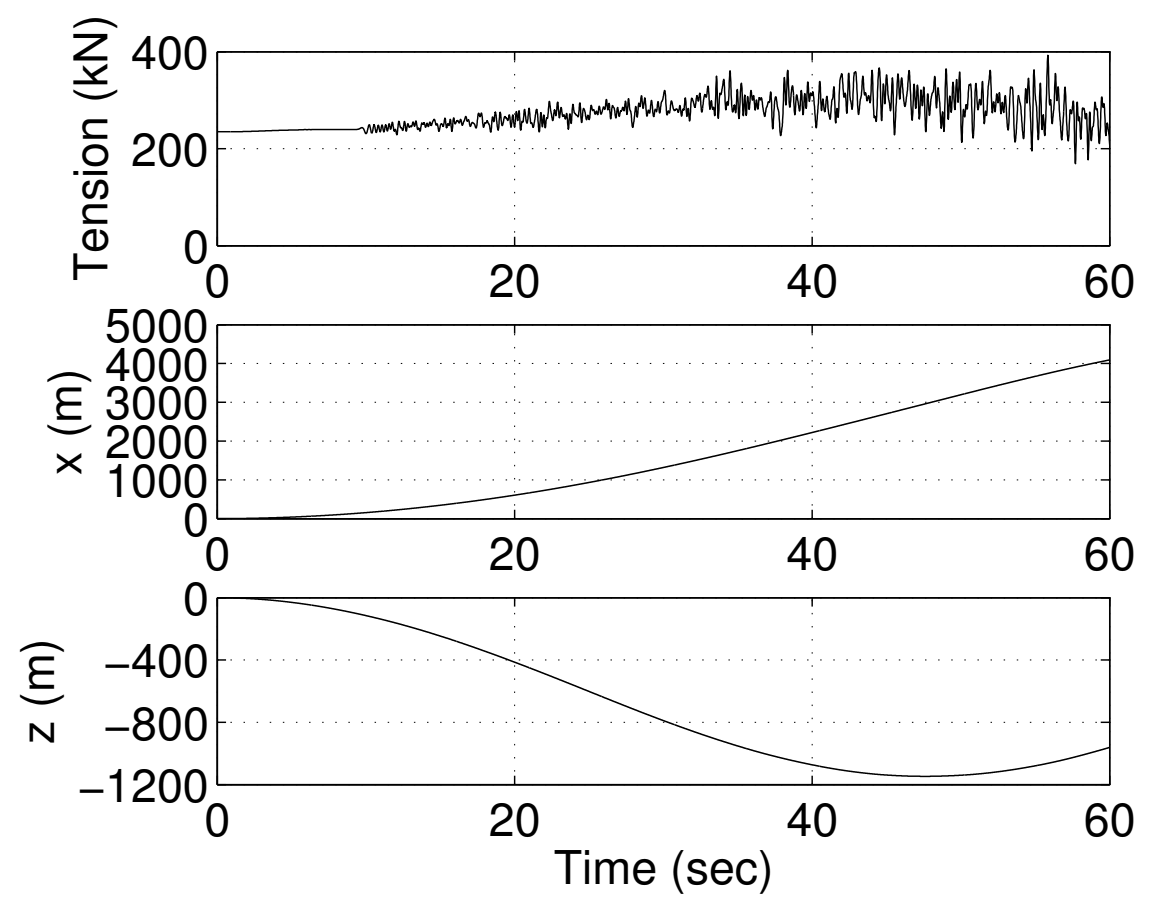

FigurE 5: (a) Tension at right support; (b) $x$-coordinate of sliding mass; (c) $z$-coordinate of sliding mass. 
Numerical results are shown in Figures 3 to 5 . Figure 3 shows a simulation of a rocket-propelled trolley with an constant thrust force applied tangential to the cable (thrust acceleration $=17.64 \mathrm{~m} / \mathrm{s}^{2}$ ). The horizontal and vertical distances of the slider correspond well with the results in [4]. There is a significant kink in the cable at the slider position, which would need a large number of modes to capture adequately using an expansion in admissible coordinates. Figure 4 shows the static equilibrium shape of the cable, together with the dynamic shape at various instants of time following the release of the slider without any thrust applied and $\mu_{k}=0.1$. Evidently the slider has a significant impact on the cable shape, mostly due to the ratio of the sliding mass to cable mass. Figure 5 shows the time history of the cable tension at the right support, as well as the in-plane coordinates of the slider. Figure 5(a) shows that the tension at the right support remains constant initially due to the time lag caused by the finite wave propagation along the cable. The motion of the slider causes significant fluctuations in the cable tension at the right support, with a maximum amplitude of $158 \mathrm{kN}$ above that of the static cable tension.

\section{Conclusions}

A dynamical model for a cable with a sliding particle was derived for general motion of the sliding mass. The model is applicable to a wide range of operating conditions and is relatively easy to implement. The method for incorporating the sliding element easily captures the kink in the cable, as well as friction effects. Numerical results show the effectiveness of the model to capture the complex cable dynamics. 


\section{References}

[1] Choo, Y. I., and Casarella, M. J., A survey of analytical methods for dynamic simulation of cable-body systems, Journal of Hydronautics, $\mathbf{7}$, 1973, 137-144. C87

[2] Wu, J. S., and Chen, C. C., The dynamic analysis of a suspended cable due to a moving load, International Journal for Numerical Methods in Engineering, 28, 1989, 2361-2381.

http://dx.doi.org/10.1002/nme.1620281011 C88

[3] Al-Qassab, M., Nair, S., and O'Leary, J., Dynamics of an elastic cable carrying a moving mass particle, Nonlinear Dynamics, 33, 2003, 11-32. http://dx.doi.org/10.1023/A:1025558825934 C88

[4] Tadjbakhsh, I. G., and Wang, Y. M., Transient vibrations of a taut inclined cable with a riding accelerating mass, Nonlinear Dynamics, 6 , 1994, 143-161. http://dx.doi.org/10.1007/BF00044982 C88, C95, C96, C99

[5] Zhou, B., Accorsi, M. L., and Leonard, J. W., Finite element formulation for modeling sliding cable elements, Computers and Structures, 82, 2004, 271-280.

http://dx.doi.org/10.1016/j.compstruc.2003.08.006 C88, C95 